\title{
Slow Conduction within Infarct Scars as a Source of High-Frequency Oscillations in the QRS Signal
}

\author{
A Kapela ${ }^{1}$, CF Starmer ${ }^{2}$, A Bezerianos ${ }^{1}$ \\ ${ }^{1}$ Department of Medical Physics, University of Patras, Greece \\ ${ }^{2}$ Department of Medicine (Cardiology), MUSC, Charleston, SC, USA
}

\begin{abstract}
We propose a detailed explanation of increased variability of high-frequency $(>100 \mathrm{~Hz})$ components within the QRS observed in post-myocardial infarction patients, reported in recent studies of real highresolution ECGs. Using mathematical models of single, branching and tortuous cardiac strands, based on cellular experimental data, we simulated effects of conduction slowing, characteristic for regions bordering infarcts, on spectral properties of the depolarization signal, assessed by means of wavelet transform and wavelet variance. Calculated extracellular potentials contained oscillations with frequencies related to propagation velocity and cellular dimensions by simple mathematical formulas. We conclude that slow AP propagation at $3-20 \mathrm{~cm} / \mathrm{s}$ within infarct scars is a possible source of high-frequency (100$300 \mathrm{~Hz}$ ) micropotentials and, with unstable activation wavefront and/or trigger jitter, contributes to the increased beat-to-beat variability in the QRS.
\end{abstract}

\section{Introduction}

In infarcted regions of cardiac ventricles the intercellular connections are distorted and the consequent discontinuous electrical activation may produce ventricular late potentials at $100-300 \mathrm{~Hz}$ with morphology and timing changing from beat to beat [1]. Possible instability in the activation wavefront can also give rise to beat-variable fractionated potentials that increase beat-to-beat variations within the QRS [2]. It was found in [3] that patients after myocardial infarction (MI) with sustained ventricular tachycardia (VT) could be distinguished from healthy persons by increased variance of the envelope and instantaneous frequency of low-frequency $12-25 \mathrm{~Hz}$ or high-frequency 120-250 Hz signals obtained from decomposition of the depolarization signals by means of the Morlet wavelet transform. According to [4], variance of the real part of complex Morlet wavelet transform of the QRS complex in $X$ lead was higher in post-myocardial infarction patients with sustained monomorphic ventricular tachycardia as compared to normals in the frequency range 200$300 \mathrm{~Hz}$. However, in those studies frequency ranges of largest discriminative power were established entirely experimentally and no theoretical justification was offered. In this work we try to identify possible sources of the beatdependent high-frequency components and explain their spectral properties. Sustained monomorphic VT after MI is usually caused by reentry facilitated through pathological regions of slow conduction. It gives necessary time for recovery of excitability of cardiac fibers in reentry circuit before next sustained excitation. Several experimental studies documented slow conduction at velocities as low as $3 \mathrm{~cm} / \mathrm{s}$ (in contrast to normal fast propagation at $50 \mathrm{~cm} / \mathrm{s}$ ) in the border zone of infarcts during the healing and healed phases [5]. We simulated three most significant types of conduction slowing characteristic for infarct scars:

- discontinuous longitudinal propagation due to increased gap-junction resistance causing intercellular electrical uncoupling [5];

- conduction through branching strands leading to currentto-load mismatch and 'pull-and-push' effect [6];

- transverse zigzag activation spread through surviving islands of myocytes interrupted by connective tissue septa [7],[8].

\section{Methods}

\subsection{Cardiac strands}

Single cable. To simulate non-homogenous propagation due to decrease in coupling conductance, we implemented one-dimensional cable with explicit gap-junction resistances [5]. Each discrete cell was composed of single sarcoplasmic reticulum and eleven isopotential segments with the LuoRudy dynamic model $L R d 00$ of the mammalian ventricular action potential [9]. The resulting PDE equation with noflux boundary conditions describing the cable was solved using the Crank-Nicholson method modified for nonuniform axial cable resistance with time resolution $\Delta t=$ $5 \mu \mathrm{s}$. To initiate cable depolarization, a current stimulus was applied to the first segment of the cable.

Branching strand. Effects of branching on excitation 

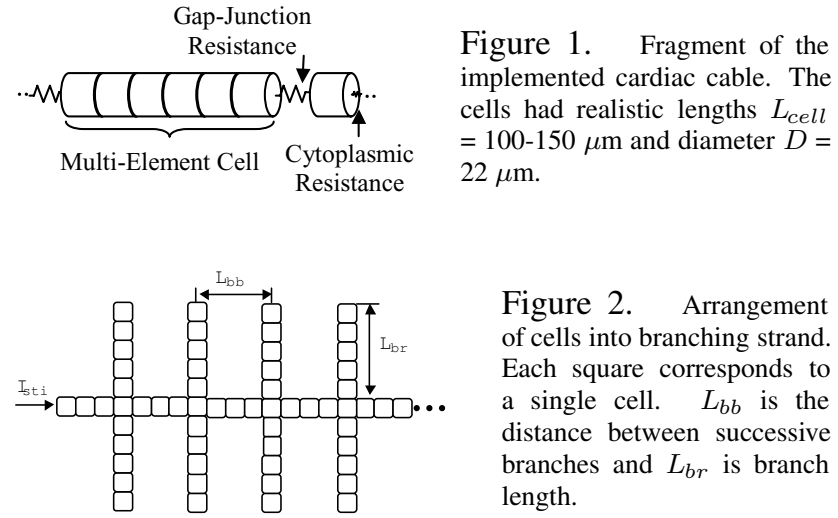

Figure 2. Arrangement of cells into branching strand. Each square corresponds to a single cell. $L_{b b}$ is the distance between successive branches and $L_{b r}$ is branch length.

spread were investigated in great detail both in experimental studies and in simulations using mathematical models [6]. We repeated the simulation using LRdO0 model with cells arranged as in fig. 2. Myoplasmic resistance was lumped with gap-junctional resistance and membrane voltage was computed using Crank-Nicholson method with spatial step $\Delta x=100 \mu \mathrm{m}$.

Tortuous route. Another important type of conduction slowing, associated with myocardial infarction and facilitating reentry, results from increased path length the activation has to travel in a matrix of merging and diverging bundles [10]. Experimental studies suggest that such activation has often form of a 'zigzag' conduction perpendicular to the fiber orientation with apparent velocities as low as $5 \mathrm{~cm} / \mathrm{s}$ [8]. Parameters $L_{b r}$ and $L_{b b}$ (see table 1) used in our simulations were mean values taken from real and more irregular tortuous routes that were determined experimentally in [7] and [8].

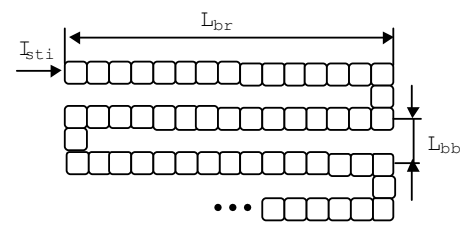

Figure 3. Tortuous arrangement of cells for computation of extracellular potentials. Total cell count - 250, number of horizontal branches 16 and 22 .

\subsection{Electrogram reconstruction}

Extracellular potentials were calculated from the formula $[11,10]$ :

$$
V_{P}(t)=\frac{\varrho D \Delta x}{4} \sum_{s} \frac{I m_{s, t}}{\sqrt{\left(x_{s}-x_{P}\right)^{2}+\left(y_{s}-y_{P}\right)^{2}}},
$$

where $V_{P}[\mu \mathrm{V}]$ is a potential at point $P\left(x_{P}\right), \operatorname{Im}\left[\mu \mathrm{A} / \mathrm{cm}^{2}\right]$ - membrane current density, $\varrho=150 \Omega \mathrm{cm}$ - specific resistivity of extracellular space. Because most of the experimental studies we refer to utilized ECG signals from
$\mathrm{X}$ lead, we calculated potential difference $U x(t)=V_{P}(t)-$ $V_{P_{0}}(t)$ with $\left(x_{P}, y_{P}\right)=(5 \mathrm{~cm}, 8.7 \mathrm{~cm}),\left(x_{P_{0}}, y_{P_{0}}\right)=(-5 \mathrm{~cm}$, $-8.7 \mathrm{~cm})$.

\subsection{The depolarization signal}

To accommodate changing morphology of fractionated potentials, we extended the mathematical model of the depolarization signal $y_{n}(t)$ used in [4] and we assumed that at $n-t h$ realization it is composed of a normal beat-independent QRS complex $U_{Q R S}(t)$ and an abnormal small-amplitude beat-dependent highfrequency oscillations $U h f_{n}(t)$ being superposition of $K=6$ components $U x_{k, n}\left(t-t_{k}-\Delta t_{k, n}\right)$ originating from different regions of infarct zone. The regions are activated with $t_{k}+\Delta t_{k, n}$ delays with respect to the QRS onset. The timing fluctuations $\Delta t_{k, n}$ are random within small interval $\left(-\Delta t_{\max }, \Delta t_{\max }\right)=(-2 \mathrm{~ms}, 2 \mathrm{~ms})$ and uncorrelated with each other, simulating unstable activation wavefront:

$$
\begin{gathered}
y_{n}(t)=U_{Q R S}(t)+U h f_{n}(t), \\
U h f_{n}(t)=\sum U x_{k, n}\left(t-t_{k}-\Delta t_{k, n}\right), \\
\operatorname{Cov}\left(\Delta T_{k}, \Delta T_{l}\right)=0, \text { for } k \neq l, \\
\Delta T_{k}=\left(\Delta t_{k, 1}, \ldots, \Delta t_{k, n}, \ldots, \Delta t_{k, N}\right) .
\end{gathered}
$$

We simulated only the $U x$ components while the $U_{Q R S}$ was modeled by Kaiser window waveform.

\subsection{Wavelet analysis}

Wavelet transformation has been successfully applied to various non-stationary physiological signals, particularly to high-resolution ECG. The wavelet transform of a nonstationary signal $y(t)$ is defined as:

$$
W T_{y}(r, t)=\frac{1}{\sqrt{r}} \int_{-\infty}^{\infty} g^{*}\left(\frac{\tau-t}{r}\right) y(\tau) d \tau,
$$

where $r$ denotes scaling factor and $g^{*}(t)$ - complex conjugate of mother wavelet (in our case Morlet wavelet).

Beat-to-beat real-part wavelet variance $\sigma_{\Re W T_{y}}^{2}(r, t)$ of $N=50$ signals $y_{n}(t)$ is calculated for each point $(r, t)$ in the time-scale plane resulting from transformation (6) according to the formula:

$$
\sigma_{\Re W T_{y}}^{2}(r, t)=\frac{1}{N} \sum_{n=1}^{N}\left[\Re W T_{y_{n}}(r, t)-\Re \overline{W T}_{y}(r, t)\right]^{2} .
$$

Combining equations (2), (3), (4), (6), (7) and using the property of linearity of the wavelet transform, we can write that:

$$
\sigma_{\Re W T_{y}}^{2}(r, t) \approx \sum_{k} \operatorname{Var}\left(\Re W T_{U x_{k}}(r, t)\right)
$$


(a)
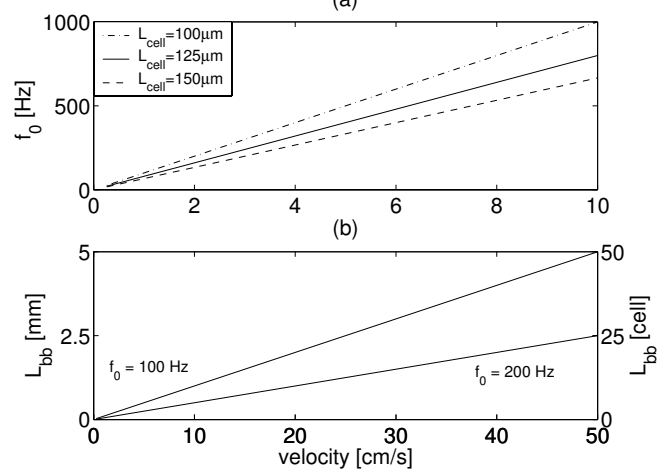

Figure 4. (a) Base frequency versus propagation velocity for different cell lengths. (b) Inter-branch distances versus velocity corresponding to $100 \mathrm{~Hz}$ and $200 \mathrm{~Hz}$ oscillations.

For a number of uncorrelated signals the wavelet variance of their superimposition is approximately the sum of their individual wavelet variances. Thus for our model the optimum frequency range for detection of the composite beat-dependent arrhythmogenic signal should include only frequencies present in individual components.

\section{Results}

Action potential propagation has been shown by many researchers both experimentally and in simulation studies based on mathematical models to be discontinuous in nature at the cellular level. Spread of excitation within a cell is very fast and continuous while at the gap junctions propagation delay occur [5]. Physiological gap-junction resistivity leads to macroscopic propagation velocity $\nu_{l}=$ $50 \mathrm{~cm} / \mathrm{s}$, the delay between excitation of consecutive cells is very small and the extracellular signal is smooth. In the case of pathologically large junctional resistivity, velocity decreases to few $\mathrm{cm} / \mathrm{s}$. Propagation delay increases to several $\mathrm{ms}$ and the cardiac cable depolarization signals $U x_{1}, U x_{2}$ contain distinct spikes (fig. 5(a)). We can calculate the base frequency corresponding to the time interval $T_{0}$ between consecutive depolarizations from the formula:

$$
f_{0}=1 / T_{0}=\nu_{l} / L_{\text {cell }}
$$

Slow conduction at $\nu_{l}=3 \mathrm{~cm} / \mathrm{s}$ gives rise to $200-300 \mathrm{~Hz}$ oscillations (fig. 4a). Figure 5(a) shows extracellular potentials $U x_{2}-U x_{4}$ produced by the branching strand with partial uncoupling during its depolarization initiated by a stimulus applied to the first cell from the left. Each period originates from depolarization of a fragment of the main strand with its corresponding branches. The base frequency corresponding to the period $T_{0}$ is:

$$
f_{0}=1 / T_{0}=\nu_{l} / L_{b b}
$$

Table 1. Parameters of the strand models

\begin{tabular}{lcccccc} 
& $U x_{1}$ & $U x_{2}$ & $U x_{3}$ & $U x_{4}$ & $U x_{5}$ & $U x_{6}$ \\
\hline$v_{l}[\mathrm{~cm} / \mathrm{s}]$ & 3 & 1.8 & 8.1 & 11.4 & 57 & 49 \\
$v_{t}[\mathrm{~cm} / \mathrm{s}]$ & - & - & - & - & 7.1 & 6.7 \\
$f_{0}[\mathrm{~Hz}]$ & 240 & 150 & 204 & 142 & 178 & 222 \\
$L_{b b}[\mu \mathrm{m}]$ & - & - & 400 & 800 & 200 & 150 \\
$L_{b r}[\mu \mathrm{m}]$ & - & - & 1500 & 800 & 1500 & 1000 \\
$R_{g}\left[\Omega \mathrm{cm}^{2}\right]$ & 200 & 300 & 38 & 30 & 1.5 & 2.5 \\
\hline
\end{tabular}

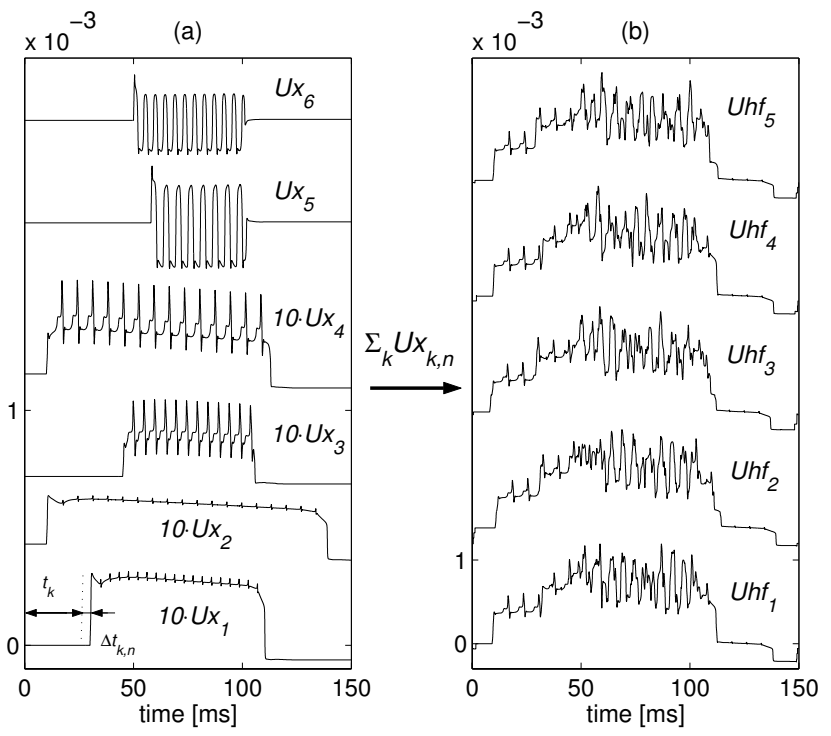

Figure 5. Summation of the signals computed from the three models of cardiac strands: (a) $U x_{1}, U x_{2}$ - potential differences from single cables, $U x_{3}, U x_{4}$ - from branching strands, $U x_{5}, U x_{6}$ - from tortuous tracts; (b) five first resultant oscillations with different morphology corresponding to different relative activation times of the individual strands. $U x_{1}-U x_{4}$ were amplified by 10 before their addition because they were significantly smaller than $U x_{5}, U x_{6}$.

where $\nu_{l}$ is mean propagation velocity along the main strand. Figure 4(b) shows relation, derived from formula (10), between mean conduction velocity and inter-branch spacing. For tortuous strands we used normal and slightly increased values of gap-junction resistance leading to longitudinal velocities $v_{l}=57 \mathrm{~cm} / \mathrm{s}$ and $v_{l}=49 \mathrm{~cm} / \mathrm{s}$. The corresponding apparent transverse velocities were $v_{t}=7.1 \mathrm{~cm} / \mathrm{s}$ and $v_{t}=6.7 \mathrm{~cm} / \mathrm{s}$. Figure 5 (a) shows the potential differences $U x_{5}, U x_{6}$ from tortuous cables. Each change in the signal polarity corresponds to the change in direction of AP propagation. The frequency corresponding to the period $T_{0}$ is $f_{0}=v_{t} / 2 L_{b b}$. Because the amplitudes of $\mathrm{AC}$ components calculated from single and branching strands were much smaller than from tortuous tracks, we suggest that the main contributor of the arrhythmogenic signal in the QRS is 'zigzag' conduction. 

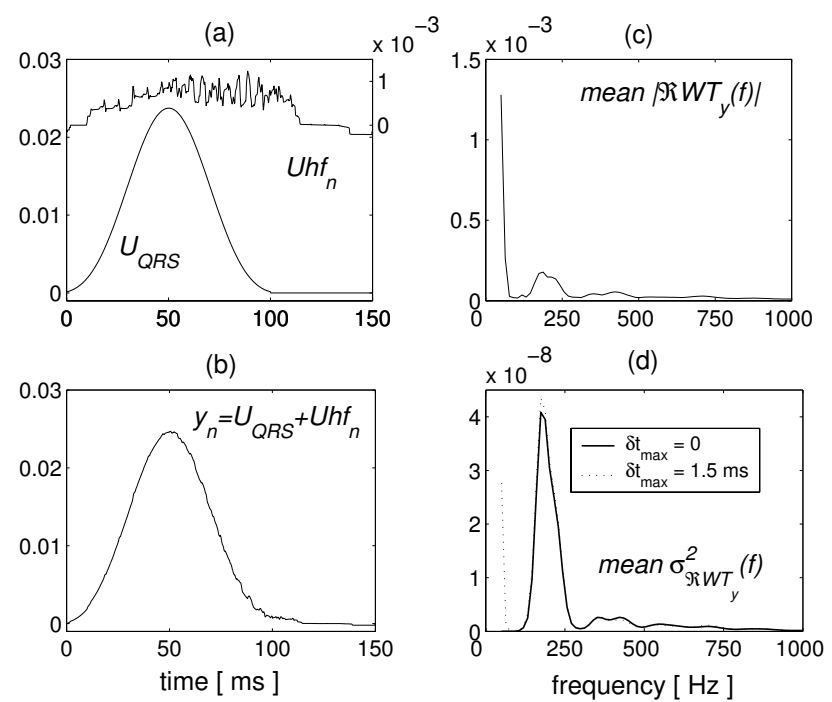

Figure 6. The QRS waveform and a high-frequency oscillation before (a) and after (b) their superimposition. (c) Mean wavelet spectrum of $50 y_{n}$ 's averaged over the QRS. (d) Wavelet variance spectrum of 50 $y_{n}$ 's averaged over the QRS. For easier interpretation the scales $r$ were transformed into corresponding frequencies; $\delta t$ - trigger jitter. Presence of the high-frequency signal in large $U_{Q R S}$ is difficult to observe in the time domain (fig. 6(b)) but is apparent after wavelet transformation (fig. 6(c)). In the idealized case of identical QRS waveform for every realization $n$ and perfect beats alignment, presence of a beat-dependent high-frequency signal is even more evident from wavelet variance than from a simple wavelet transform of a single beat because of the overlapping spectrum of the large QRS signal (compare fig. 6(c) and 6(d))

\section{Discussion and conclusions}

In general, other conditions associated with conduction slowing and arrhythmia, like ischemia and cardiac sodium channel mutations [12], can also give rise to a highfrequency signal if superimposed on a highly fibrotic region. On the other hand, fast propagation and sparse branching, theoretically able to produce $100 \mathrm{~Hz}-200 \mathrm{~Hz}$ (see fig. 4(b)), is unlikely to contribute many periods in the QRS signal due to need to travel long distance of several centimeters to last $100 \mathrm{~ms}$ (QRSduration. velocity $=100 \mathrm{~ms} \cdot 50 \mathrm{~cm} / \mathrm{s}=5 \mathrm{~cm}$ ). This study was motivated mainly by results and methods presented in [4]. We aimed at providing a link between electrophysiological changes and properties of the QRS signal. Until now the assessment of the VT risk in post-MI patients by wavelet transform variance was based on putative frequency characteristics of the arrhythmogenic signals due to lack of their mathematical description. In this study we propose a simple mathematical relations between cellular dimensions, conduction velocity and frequency spectrum of depolarization signal, able to explain some of the experimental data. We conclude that slow AP propagation through fibrotic regions after MI is a likely source of the high-frequency $(100-300 \mathrm{~Hz})$ fractionated micropotentials and together with beat-dependent activation delays gives rise also to the variability of high-frequency components within the QRS.

\section{References}

[1] Zimmermann M, Adamec R, Simonin P, Richez J. Beatto-beat detection of ventricular late potentials with high resolution electrocardiography. Am Heart J 1991;121:57685.

[2] Shaw G, Savard P. On the detection of QRS variations in the ECG. IEEE Trans on Biomed Eng 1995;42:736-41.

[3] Link A, Endt P, Oeff M, Lutz T. Variability of the QRS signal in high-resolution electrocardiograms and magnetocardiograms. IEEE Trans on Biomed Eng 2001; 48:133-42.

[4] Popescu M, Laskaris N, Chiladakis I, Stathopoulos C, Cristea P, Manolis A, Bezerianos A. Beat-to-beat wavelet variance of the QRS complex as a marker of arrhythmogenic substrate in ventricular tachycardia patients. Pysiol Meas 1998;19:77-92.

[5] Rudy Y. Models of continuous and discontinuous propagation in cardiac tissue. In Zipes DP (ed.), Cardiac electrophysiology - from cell to bedside, second edition. W. B. Saunders Company, 1995; 326-34.

[6] Kucera JP, Rudy Y. Mechanistic insights into very slow conduction in branching cardiac tissue. A model study. Circ Res 2001;89:799-806

[7] de Bakker JMT, van Capelle FJL, Janse MJ, Tasseron S, Vermeulen JT, de Jonge N, R LJ. Slow Conduction in the Infarcted Human Heart. 'Zigzag' Course of Activation. Circulation 1993;88:915-26.

[8] Gardner PI, Ursell PC, Fenoglio JJ, Wit AL. Electrophysiologic And Anatomic Basis for Fractionated Electrograms Recorded From Healed Myocardial Infarcts. Circulation 1985;72(3):596-611.

[9] Faber GM, Rudy Y. Action potential and contractility changes in $[\mathrm{Na}(+)](\mathrm{i})$ overloaded cardiac myocytes: a simulation study. Biophys J 2000;78:2392-404.

[10] Maglaveras N, De Bakker JM, Van Capelle FJ, Pappas C, Janse MJ. Activation delay in healed myocardial infarction: a comparison between model and experiment. Am J Physiol 1995;269:H1441-9.

[11] Cimponeriu A, Starmer CF, Bezerianos A. A theoretical analysis of acute ischemia and infarction using ECG reconstruction on a 2-D model of myocardium. IEEE Trans on Biomed Eng 2001;48(1):41-54.

[12] Starmer CF, Colatsky TJ, Grant AO. What happens when cardiac Na channels lose their function? 1 Numerical studies of the vulnerable period in tissue expressing mutant channels. Cardiovascular Research 2003;57:82-91.

Address for correspondence:

Anastasios Bezerianos, email: bezer@patreas.upatras.gr,

Dept. of Medical Physics, School of Medicine,

University of Patras,26500 Rion-Patras, Greece. 